\title{
Seite der Therapie - Page de thérapeutique
}

Cuendet, A.: A propos de la cholécystectomie d'urgence dans la cholécystite aiguë. Méd. et Hyg. 15: 247-248, 30 mai 1957.

C'est sur Гétude d'une statistique de 91 cas que Tauteur fonde sa ligne de conduite dont il expose les principes: malgré les résultats immédiats favorables de la thérapeutique médicale, Tintervention d'urgence est indiquée, si toutefois l'état general le permet. C'est dans les trois premiers jours que les conditions opératoires et le pronostic seront les meilleurs. En revanche, la cholécystectomie retardée (5e-15e jour) est grevée d'une mortalité importante.

Si une lithiase cholédocienne est suspectée, la cure chirurgicale sera différée, aìm de permettre, à froid, une exploration soigneuse de la voie biliaire principale, manceuvre peu compatible avec les conditions de $\Gamma$ urgence. Le traitement medical n'est souvent qu'un palliatif: la récidive est fréquente, qui conduit en dernier ressort le malade au chirurgien. $\mathrm{Ph}$ Secretan Jadot, R. et Lafontaíne, A.: Intoxication par le sous-nitrate de bismuth chez un gaslrectomisé. Acta gastro-ent. belg. 20: 629-639 (juill. 1957).

L'intoxication, du type nitritoïde, par le sous-nitrate de bismuth, est aussi classique qu'exceptionnelle. L'observation rapportée ici concerne un ulcéreux, opéré 4 fois, sans succès durable, habitue depuis 7 ans au bismuth à hautes doses, et qui, après une vagotomie pour ulcère peptique jéjunal présente une cyanose bleu foncé, avec inconscience, mais sans symptômes circulatoires ou respiratoires alar-mants. On pose le diagnostic d'intoxication par poison méthémoglobinisant, et le traitement avec des oxydants rétablit la situation en quelques heures. La flore intestinale de ce malade, composée essentiellement de bac. proteus, fer-mentait avec acidification, était douée d'un fort pouvoir réducteur, et cultivée in vitro avec du sous-nitrate de bismuth donnait un dégagement de No 2. Ces considerations n'expliquent toutefois pas Tapparition subite d'une intoxication chez un malade habitue au bismuth à hautes doses depuis de nombreuses années.

20 Gastroentei-ologia, Vol. 89, No. 3/4 (1958) 